\title{
Maternal and fetal outcomes in pregnant Japanese women with inflammatory bowel disease: our experience with a series of 23 cases
}

\author{
Naoki Minami ${ }^{1}$, Minoru Matsuura ${ }^{1}$, Yorimitsu Koshikawa ${ }^{1}$, Satoshi Yamada ${ }^{1}$, Yusuke Honzawa ${ }^{1}$, Shuji \\ Yamamoto $^{1}$, Hiroshi Nakase ${ }^{2}$ \\ ${ }^{1}$ Department of Gastroenterology and Hepatology, Graduate School of Medicine Kyoto University, Kyoto, ${ }^{2}$ Department of Gastroenterology and \\ Hepatology, Sapporo Medical University School of Medicine, Sapporo, Japan
}

Background/Aims: Our physicians work to expand the possibilities to treat female patients with inflammatory bowel disease (IBD) who wish to become pregnant. Although many drugs, including 5-aminosalicylate (5-ASA), corticosteroids, immunomodulators, and biologics, are used safely during pregnancy, few reports have described the therapeutic regimen throughout pregnancy and the management of patients who relapse during pregnancy precisely. The aim of this study was to assess the management of patients with IBD during pregnancy. Methods: We identified 19 patients (five with Crohn's disease and 14 with ulcerative colitis [UC]) who became pregnant with a total of 23 pregnancies between May 2005 and May 2015 by reviewing the medical records of Kyoto University Hospital. The following data were collected: the maternal variables, the IBD treatment type, the disease activity, the pregnancy outcome, and the mode of delivery. Results: Among the 19 patients, 18 had become pregnant after being diagnosed with IBD, while one had developed UC newly after pregnancy. Throughout the gestation, all patients were treated with probiotics, 5-ASA, prednisolone, cytapheresis, or infliximab. The relapse rate during pregnancy was $21.7 \%$ (5/23 cases). The five patients who experienced a relapse were able to pursue their pregnancy after intensification of their treatments. There were no adverse fetal or neonatal problems, except in one case that required an emergency Caesarean section because of placental dysfunction and in which a very low-birth-weight infant was born preterm. Conclusions: Our present data confirmed that even if the disease flares up during pregnancy, good pregnancy outcomes can be achieved with an optimal intensification of the patient's treatment. (Intest Res 2017;15:90-96)

Key Words: Inflammatory bowel disease; Pregnancy; Therapeutic management

Received August 1, 2016. Revised August 31, 2016.

Accepted September 5, 2016.

Correspondence to Hiroshi Nakase, Department of Gastroenterology and Hepatology, Sapporo Medical University School of Medicine, S-1, W-16, Chuo-ku, Sapporo 060-8543, Japan. Tel: +81-11-611-2111, Fax: +81-11613-1241, E-mail: hiropynakase@gmail.com

Financial support: This work was supported by the Japan Society for the Promotion of Science (JSPS)'s Grants-in-Aid for Scientific Research (24590941, 26460967, and 16K19150), by the Health and Labor Sciences Research Grants for Research on Rare and Intractable Diseases of the Ministry of Health, Labor, and Welfare of Japan, and by the Japan Foundation for Applied Enzymology. Conflict of interest: None.

\section{INTRODUCTION}

Inflammatory bowel disease (IBD), which comprises CD and UC, is an idiopathic chronic intestinal disorder. ${ }^{1}$ As the peak onset of IBD is in the second and third decades of life, ${ }^{2}$ most female patients with IBD will experience pregnancy and delivery during the clinical course. Therefore, when treating female patients with IBD who wish to become pregnant, physicians must consider the issue of fertility. Moreover, when managing patients, physicians must keep in mind both, the disease activity and the growth of the fetus after the patient becomes pregnant.

\footnotetext{
๑ Copyright 2017. Korean Association for the Study of Intestinal Diseases. All rights reserved.

This is an Open Access article distributed under the terms of the Creative Commons Attribution Non-Commercial License (http://creativecommons.org/licenses/by-nc/4.0)

which permits unrestricted non-commercial use, distribution, and reproduction in any medium, provided the original work is properly cited.
} 
Many drugs, including 5-aminosalicylate (5-ASA), corticosteroids, immunomodulators, and biologics, are being used to treat IBD. ${ }^{3}$ According to the guidelines of the European Crohn's and Colitis Organization (ECCO), ${ }^{4}$ these drugs can be used safely during pregnancy. However, few reports have described the therapeutic regimen throughout pregnancy and the management of patients who relapse during pregnancy precisely. Herein, we discuss the management of patients with IBD during pregnancy based on 23 cases we observed.

\section{METHODS}

\section{Patients}

The research was conducted as a single-center retrospective cohort study. We reviewed the medical records of all female patients with IBD who had been treated and followed up by the Kyoto University Hospital between May 2005 and May 2015. A total of 170 female patients with IBD (46 with CD and 124 with UC) were enrolled. Of the 170 female IBD patients, we identified 19 patients (five with CD and 14 with UC) who had become pregnant with a total of 23 pregnancies over the study period. Of the 19 patients, 18 had become pregnant after being diagnosed with IBD and were treated with probiotics, 5-ASA, or infliximab (IFX) when the pregnancy was confirmed. Only one patient had developed UC newly after pregnancy. Moreover, all the patients were treated with probiotics, 5-ASA, prednisolone (PSL), cytapheresis, or IFX throughout the gestation. This retrospective singlecenter study was conducted in accordance with the principles of the Declaration of Helsinki, and was reviewed and approved by the Institutional Review Board of Kyoto University Hospital.

\section{Data Acquisition}

The following data were collected: (1) the maternal variables, (2) the IBD treatment type, (3) the disease activity, (4) the pregnancy outcome, and (5) the mode of delivery. The maternal variables included the age at conception, the number of previous pregnancies, and details of the underlying IBD. The therapeutic regimen and disease activity were recorded at conception and every third month thereafter. To evaluate the disease activity, the Simple Clinical Colitis Activity Index (SCCAI) ${ }^{5}$ was used for UC patients, and Harvey \& Bradshaw's activity index (HBI $)^{6}$ was used for CD patients. Each trimester, we classified the disease activity into two categories: remission (SCCAI $\leq 3$ and $\mathrm{HBI} \leq 5$ ) or active disease. ${ }^{7}$

\section{RESULTS}

\section{Patients' Baseline Characteristics}

We identified 23 pregnancies in 19 patients with IBD (four patients experienced two pregnancies) between May 2005 and May 2015. All the patients had become pregnant during clinical remission (CR). The maternal variables are shown in Table 1. The mean age of the 23 patients at pregnancy was 32.9 years (range, 27-43 years). Among the 14 patients with UC, five had pancolitis, seven had left-sided colitis, one had proctitis, and one had proctosigmoiditis. Of the five patients with $\mathrm{CD}$, two presented small bowel involvement, and three presented small bowel and colon involvement. Four CD cases and one UC case had a history of intestinal surgery before becoming pregnant. Four UC cases and four CD cases had a history of one previous delivery.

\section{Pregnancy Outcome and Mode of Delivery}

The mean gestation at delivery was 38.8 weeks (range, 30-41 weeks). Case 10 required an emergency Caesarean section due to placental dysfunction caused by gestational hypertension, and delivered preterm despite maintaining CR throughout the pregnancy. Although a very low-birthweight infant was born as a result, no congenital malformations were found. Five other cases also required Caesarean sections for the following reasons: a history of uterine myomectomy in case 1, breech presentation in cases 9 and 13, fetal distress in case 16, and a history of previous Caesarean section in case 17 . The remaining 17 cases gave birth by spontaneous vaginal delivery. Although case 20 had a history of anal lesion treated with a seton placement, she was able to give birth by spontaneous vaginal delivery. The median neonatal birth weight was 2,992 $\mathrm{g}$ (range, 1,174-3,790 g). All of the infants, except for those in cases 10 and 16, were over 2,500 g. Although the disease activity in the mother had been well controlled with 3,000 mg 5-ASA/day, and despite being full-term, the infant in case 16 was under 2,500 g. No congenital malformation was observed in any of the infants. Moreover, none of the patients followed throughout the observational period had a miscarriage (Table 2).

\section{Treatment for IBD and Disease Activity throughout Pregnancy}

In the first trimester, 22 of the 23 cases maintained CR. The remaining case (case 15) developed UC newly dur- 
Table 1. Patients' Characteristics with Maternal Variables

\begin{tabular}{|c|c|c|c|c|c|c|}
\hline Patient & Case & Diagnosis & Age (yr) & Disease location & Previous surgery & $\begin{array}{c}\text { No. of previous } \\
\text { pregnancies }\end{array}$ \\
\hline 1 & Case 1 & UC & 36 & Left-sided colitis & None & 0 \\
\hline 2 & Case 2 & UC & 34 & Extensive colitis & None & 0 \\
\hline 3 & Case 3 & UC & 35 & Left-sided colitis & None & 0 \\
\hline 4 & Case 4 & UC & 34 & Extensive colitis & None & 0 \\
\hline 5 & Case 5 & UC & 32 & Proctitis & None & 0 \\
\hline 6 & Case 6 & UC & 35 & Left-sided colitis & None & 1 \\
\hline 7 & Case 7 & UC & 35 & Extensive colitis & None & 1 \\
\hline 8 & Case 8 & UC & 35 & Left-sided colitis & None & 0 \\
\hline 9 & $\begin{array}{l}\text { Case } 9 \\
\text { Case } 10\end{array}$ & UC & $\begin{array}{l}33 \\
36\end{array}$ & Left-sided colitis & $\begin{array}{l}\text { None } \\
\text { None }\end{array}$ & $\begin{array}{l}0 \\
1\end{array}$ \\
\hline 10 & Case 11 & UC & 29 & Extensive colitis & None & 1 \\
\hline 11 & Case 12 & UC & 31 & Proctosigmoiditis & None & 0 \\
\hline 12 & Case 13 & UC & 34 & Extensive colitis & None & 0 \\
\hline 13 & Case 14 & UC & 29 & Left-sided colitis & None & 0 \\
\hline 14 & Case 15 & UC & 43 & Left-sided colitis & Appendectomy & 0 \\
\hline 15 & $\begin{array}{l}\text { Case } 16 \\
\text { Case } 17\end{array}$ & $C D$ & $\begin{array}{l}27 \\
29\end{array}$ & Small bowel & $\begin{array}{l}\text { None } \\
\text { Small bowel resection }\end{array}$ & $\begin{array}{l}0 \\
1\end{array}$ \\
\hline 16 & $\begin{array}{l}\text { Case } 18 \\
\text { Case } 19\end{array}$ & $C D$ & $\begin{array}{l}28 \\
30\end{array}$ & Small bowel & Small bowel resection & $\begin{array}{l}0 \\
1\end{array}$ \\
\hline 17 & Case 20 & $C D$ & 33 & Small bowel+colon & Ileocecal resection, seton & 1 \\
\hline 18 & Case 21 & $C D$ & 36 & Small bowel+colon & None & 0 \\
\hline 19 & $\begin{array}{l}\text { Case } 22 \\
\text { Case } 23\end{array}$ & $C D$ & $\begin{array}{l}30 \\
33\end{array}$ & Small bowel+colon & Small bowel resection & $\begin{array}{l}0 \\
1\end{array}$ \\
\hline
\end{tabular}

ing the first trimester and showed mild disease activity. Of the 23 cases, 12 were treated with 5-ASA, four with 5-ASA and IFX, one with IFX, and six with probiotics. The strain of probiotics used in the three UC cases (cases 7, 13, and 15) was Bifidobacterium bifidum (Biofermin tablets). Among the three cases that received probiotics as a maintenance therapy, cases 7 and 13 had been treated with thiopurines in addition to probiotics before pregnancy, as they were allergic to 5-ASA. However, they stopped taking thiopurines after finding out about their pregnancy, as they feared the drug's potential side effects on the fetus. Therefore, they received probiotics alone throughout the pregnancy. Despite her mild disease activity, case 15 was treated with probiotics due to her allergy to 5-ASA. As patient 19 had undergone bowel resection in 2003, before immunomodulators and anti-tumor necrosis factor (anti-TNF) agents had been approved for the treatment of CD in Japan, she had been treated with an elemental diet, 5-ASA, and probiotics after the surgery. She had remained in remission without either immunomodulators or anti-TNF agents. Fortunately, remission was maintained with probiotics alone after she stopped her elemental diet and the 5-ASA, and she experienced two pregnancies without trouble.

In the second trimester, the disease flared up in four of the 23 cases (1, 9, 11, and 15), calling for intensification of the patients' treatments. CR was achieved by weekly cytapheresis in case 1. In case 9, both cytapheresis and $20 \mathrm{mg}$ PSL/ day were used. CR was achieved with an escalating dose of 5-ASA from 2,000 mg/day to 4,000 mg/day in case 11 , and by induction treatment with $25 \mathrm{mg}$ PSL/day in case 15 .

In the third trimester, case 8 experienced a flare-up. An increased dose of IFX from $7.5 \mathrm{mg} / \mathrm{kg}$ to $10 \mathrm{mg} / \mathrm{kg}$ every 4 weeks improved her symptoms, resulting in the delivery of a healthy baby. In all cases except for those in which the disease flared up, the therapeutic regimen was not changed throughout the pregnancy. For the cases treated with 5-ASA the mean dose of 5-ASA was 2,769 mg/day (range, 2,000$4,000 \mathrm{mg} /$ day). None of the pregnant patients was treated 
Table 2. Pregnancy Outcomes and Mode of Delivery

\begin{tabular}{ccccccc}
\hline Patient & Case & Diagnosis & Age (yr) & Gestation at delivery (wk) & Mode of delivery & Neonatal birth weight (g) \\
\hline 1 & Case 1 & UC & 36 & NA & C-section & NA \\
2 & Case 2 & UC & 34 & 40 & VD & VD 370 \\
3 & Case 3 & UC & 35 & NA & VD & NA \\
4 & Case 4 & UC & 34 & 41 & VD & 3,488 \\
5 & Case 5 & UC & 32 & 41 & VD & NA \\
6 & Case 6 & UC & 35 & NA & VD & NA \\
7 & Case 7 & UC & 35 & NA & VD & 2,638 \\
8 & Case 8 & UC & 35 & 39 & C-section & 2,680 \\
9 & Case 9 & UC & 33 & 37 & C-section & 1,174 \\
& Case 10 & & 36 & 30 & VD & 3,185 \\
10 & Case 11 & UC & 29 & 39 & C-section & 2,715 \\
11 & Case 12 & UC & 31 & 39 & VD & 3,424 \\
12 & Case 13 & UC & 34 & 38 & VD & 3,530 \\
13 & Case 14 & UC & 29 & 39 & C-section & 2,710 \\
14 & Case 15 & UC & 43 & 37 & C-section & 2,376 \\
15 & Case 16 & CD & 27 & 39 & VD & 2,602 \\
& Case 17 & & 29 & 41 & VD & 3,134 \\
16 & Case 18 & CD & 28 & 39 & VD & 2,962 \\
& Case 19 & & 30 & 38 & VD & NA \\
17 & Case 20 & CD & 33 & NA & VD & NA \\
18 & Case 21 & CD & 36 & NA & 41 & 3,638 \\
19 & Case 22 & CD & 30 & 41 & 3,790 \\
\hline
\end{tabular}

$\mathrm{NA}$, not applicable; $\mathrm{C}$-section, Caesarean section; VD, vaginal delivery.

with thiopurine or calcineurin inhibitors (Table 3).

\section{DISCUSSION}

In this retrospective cohort study, the IBD relapse rate during pregnancy was $21.7 \%$ ( $5 / 23$ cases). The five cases that experienced a relapse were able to continue their pregnancy with an intensification of their treatments. In addition, there were no adverse fetal or neonatal problems except in case 10, in which an emergency Caesarean section was required on account of placental dysfunction, and a very low-birthweight infant was born preterm.

According to the ECCO guidelines, ${ }^{4}$ the safety of treatment with 5-ASA during pregnancy is acceptable. In our study, 16 of 23 cases were administered 5-ASA throughout their pregnancy, at a mean dose of 2,769 mg/day (range, 2,000-4,000 $\mathrm{mg}$ /day). With the exception of case 11, in which the patient experienced a UC flare-up in the second trimester, the same dose of 5-ASA was maintained throughout the pregnancy. In the cases treated with 5-ASA, no major fetal adverse events, such as stillbirth or congenital abnormality, occurred. Although a low-birth-weight infant was born despite the fullterm gestation in case 16, it remains unclear whether the 5-ASA affected the pregnancy outcome, as the disease activity of the mother had been well controlled with 3,000 mg of 5-ASA/day and she had no underlying disorder except for stable CD.

According to the ECCO guidelines, ${ }^{4}$ the use of corticosteroids in pregnant patients with IBD is also acceptable. In particular, PSL is less likely to affect the fetus due to its inactivation by placental $11 \beta$-hydroxy steroid dehydrogenase. ${ }^{8}$ In our study, two cases (9 and 15) were treated with PSL from the second to the third trimester due to flare-ups, and the use of PSL did not affect the pregnancy outcomes.

The use of IFX in the third trimester is not recommended, as it can pass through the placental barrier at this time and 


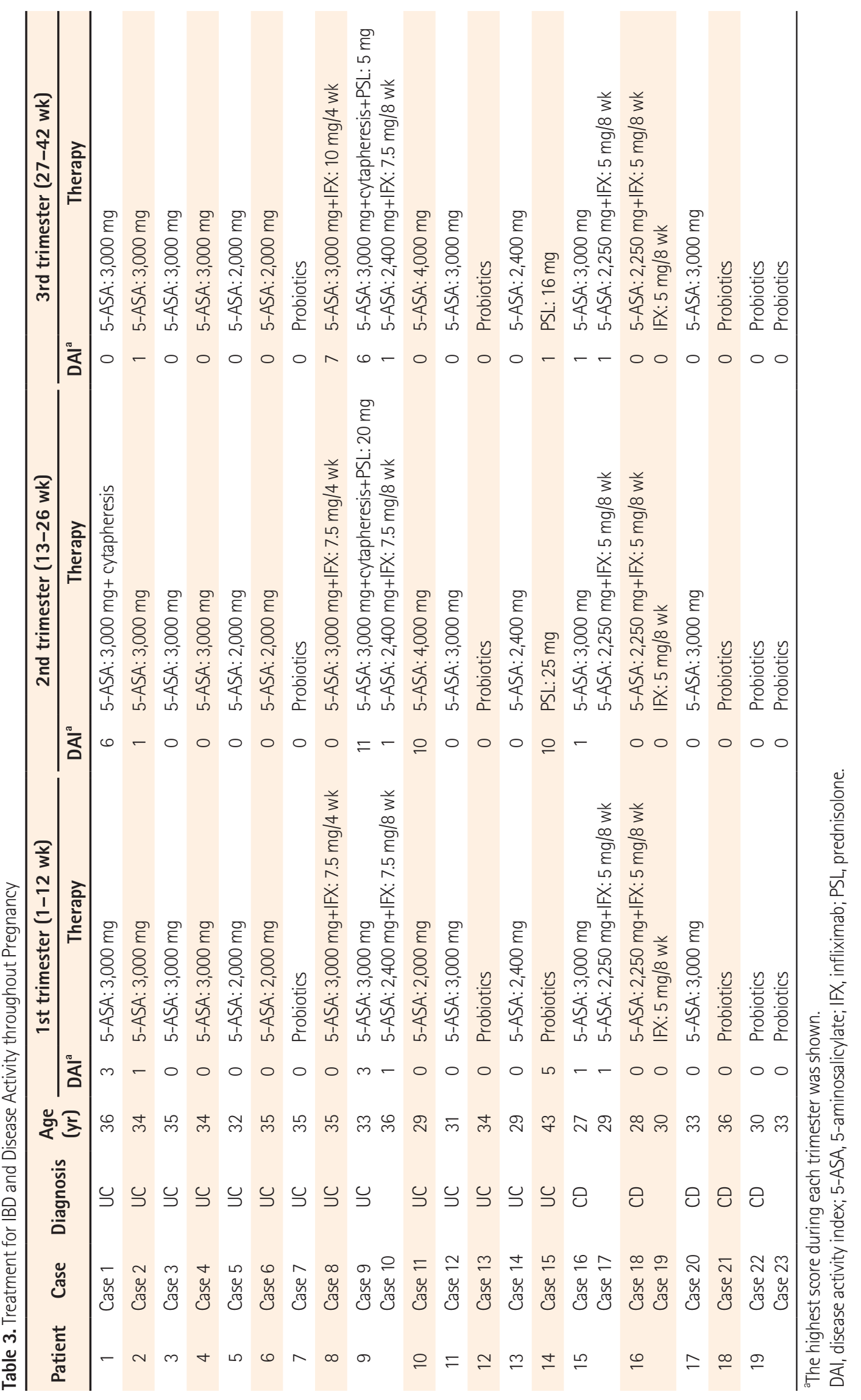


can affect the fetus and newborn., ${ }^{4,9}$ The use of IFX beyond the second trimester raises concerns about the infant's immune function, as the drug has been detected in infants up to 6 months after birth. ${ }^{10}$ In these infants, vaccinations with live vaccines must be avoided before 6 months after birth. ${ }^{4}$ In this study, we maintained the use of IFX in the third trimester to avoid flare-ups in refractory cases. Fortunately, there were no adverse effects on the fetuses or newborns. Zelinkova et al. ${ }^{11}$ reported that the intentional discontinuation of anti-TNF agents toward the end of the second trimester did not increase the risk of IBD flare-ups during pregnancy, although the study was only based on a small number of cases. Therefore, in cases of remission, the intentional discontinuation of IFX before the third trimester should be considered. However, more data about pregnant patients who stop taking biologics are needed. A recent multicenter study conducted in Japan reported that the increased spontaneous abortion rate might be associated with anti-TNF use. ${ }^{12}$ In our study, there was no case of spontaneous abortion regardless of IFX use. However, a further large-scale study is needed to elucidate the exact relationship between spontaneous abortion and IBD treatments.

Cytapheresis, which was originally devised in Japan, ${ }^{13}$ is considered to be safe for pregnant patients, as it does not involve drugs that might affect the fetus. Actually, there have been a few reports of cytapheresis being effective in pregnant IBD patients, with no adverse events to their fetuses. ${ }^{14,15}$ In two cases of flare-ups in the present study (cases 1 and 9), the disease activity was reduced by cytapheresis, with no adverse effects on the fetuses. Therefore, cytapheresis may be one of the options to consider before the initiation of treatment with immunosuppressants in cases of flare-ups during pregnancy, although evidence from more cases is needed.

A European cohort study revealed no difference in the flare rates between pregnant IBD patients and non-pregnant IBD patients who had become pregnant during remission. ${ }^{16}$ Moreover, a recent prospective European case-control study also revealed a low relapse rate throughout pregnancy (14.2\% in CD patients and $26.4 \%$ in UC patients), as the majority of the IBD patients (86.9\% with CD and $79 \%$ with UC) had been in remission at conception and during the first trimester. ${ }^{7}$ According to these studies, the maintenance of CR before conception is the first step in the management of IBD patients who want to become pregnant. In our study, the relapse rate $(21.7 \%)$ during pregnancy was similar to that in the European case-control study, since the pregnant IBD patients were also able to maintain CR before conception.

Among the 23 cases, only one fetal developmental de- fect-caused by placental dysfunction-occurred (case 10). In this case, we did not consider that the UC had affected the fetal development, as the disease activity had been well controlled throughout the pregnancy.

The main limitation of this study lies in its absence of documentation of the maternal and fetal outcomes of patients taking thiopurines during pregnancy. In three cases $(7,8$, and 13$)$, patients who had been receiving azathioprine stopped taking it before conception as they were worried about the effects of the thiopurines on their fetus. Fortunately, they experienced no flare-up during pregnancy. In a Cancers Et Surrisque Associé aux Maladies inflammatoires intestinales En France cohort study, ${ }^{17}$ the use of thiopurines during pregnancy did not increase the risk of neonatal congenital abnormalities. Conversely, a recent prospective study demonstrated that $60 \%$ of all infants exposed to maternal thiopurine use during pregnancy presented anemia at birth. ${ }^{18}$ Therefore, there are still many unknowns about the safety of thiopurines for both fetuses and infants. In clinical practice, many Japanese female patients with IBD prefer to stop taking thiopurines before conception, as was observed in this study. However, it should always be kept in mind that the avoidance of IBD flare-ups ultimately brings better pregnancy outcomes. To this date, there have been several reports on the association between the withdrawal of thiopurines from UC patients and high relapse rates. ${ }^{19-21}$

A recent report by Moreno-Rincón et $\mathrm{al}^{21}{ }^{21}$ suggested that the relapse rate after withdrawal from thiopurines depended on the duration of the thiopurine treatment. Therefore, thiopurine cessation should not be decided automatically based on the patient's wish only. To solve this issue, in the future, it is necessary to identify the subpopulation of female patients with IBD who might be able to maintain remission even after thiopurine withdrawal.

In conclusion, it is important for IBD patients who wish to become pregnant to maintain CR before conception in order to avoid a flare-up during pregnancy. Moreover, good pregnancy outcomes are expected with an optimal intensification of the treatment if the disease flares up. As the number of pregnant IBD patients continues to increase in Japan and globally, the establishment of guidelines for the best possible management of female IBD patients during pregnancy is urgently needed.

\section{ACKNOWLEDGEMENTS}

N.M., M.M., and H.N. designed the study. N.M. performed the major roles of patients' data collection and manuscript- 
writing. Y.K., S.Y., Y.H., S.Y., M.M., and H.N. collected the patient's data. H.N. was also involved in the editing of the manuscript. All authors read and approved the final manuscript.

\section{REFERENCES}

1. Abraham C, Cho JH. Inflammatory bowel disease. N Engl J Med 2009;361:2066-2078.

2. Loftus EV Jr, Sandborn WJ. Epidemiology of inflammatory bowel disease. Gastroenterol Clin North Am 2002;31:1-20.

3. Baumgart DC, Sandborn WJ. Inflammatory bowel disease: clinical aspects and established and evolving therapies. Lancet 2007;369:1641-1657.

4. van der Woude CJ, Ardizzone S, Bengtson MB, et al. The second European evidenced-based consensus on reproduction and pregnancy in inflammatory bowel disease. J Crohns Colitis 2015;9:107-124.

5. Walmsley RS, Ayres RC, Pounder RE, Allan RN. A simple clinical colitis activity index. Gut 1998;43:29-32.

6. Harvey RF, Bradshaw JM. A simple index of Crohn's-disease activity. Lancet 1980;1:514.

7. Bortoli A, Pedersen N, Duricova D, et al. Pregnancy outcome in inflammatory bowel disease: prospective European casecontrol ECCO-EpiCom study, 2003-2006. Aliment Pharmacol Ther 2011;34:724-734.

8. Vermeire S, Carbonnel F, Coulie PG, et al. Management of inflammatory bowel disease in pregnancy. J Crohns Colitis 2012;6:811-823.

9. Kane SV, Acquah LA. Placental transport of immunoglobulins: a clinical review for gastroenterologists who prescribe therapeutic monoclonal antibodies to women during conception and pregnancy. Am J Gastroenterol 2009;104:228-233.

10. Vasiliauskas EA, Church JA, Silverman N, Barry M, Targan SR, Dubinsky MC. Case report: evidence for transplacental transfer of maternally administered infliximab to the newborn. Clin Gastroenterol Hepatol 2006;4:1255-1258.
11. Zelinkova Z, van der Ent C, Bruin KF, et al. Effects of discontinuing anti-tumor necrosis factor therapy during pregnancy on the course of inflammatory bowel disease and neonatal exposure. Clin Gastroenterol Hepatol 2013;11:318-321.

12. Komoto S, Motoya S, Nishiwaki Y, et al. Pregnancy outcome in women with inflammatory bowel disease treated with antitumor necrosis factor and/or thiopurine therapy: a multicenter study from Japan. Intest Res 2016;14:139-145.

13. Sawada K, Ohnishi K, Fukui S, et al. Leukocytapheresis therapy, performed with leukocyte removal filter, for inflammatory bowel disease. J Gastroenterol 1995;30:322-329.

14. Okada H, Makidono C, Takenaka R, et al. Therapeutic efficacy of leukocytapheresis in a pregnant woman with severe active ulcerative colitis. Digestion 2006;74:15-18.

15. Takahashi H, Sugawara K, Sugimura M, et al. Flare up of ulcerative colitis during pregnancy treated by adsorptive granulocyte and monocyte apheresis: therapeutic outcomes in three pregnant patients. Arch Gynecol Obstet 2013;288:341-347.

16. Riis L, Vind I, Politi P, et al. Does pregnancy change the disease course? A study in a European cohort of patients with inflammatory bowel disease. Am J Gastroenterol 2006;101:1539-1545.

17. Coelho J, Beaugerie L, Colombel JF, et al. Pregnancy outcome in patients with inflammatory bowel disease treated with thiopurines: cohort from the CESAME study. Gut 2011;60:198-203.

18. Jharap B, de Boer NK, Stokkers P, et al. Intrauterine exposure and pharmacology of conventional thiopurine therapy in pregnant patients with inflammatory bowel disease. Gut 2014;63:451-457.

19. Hawthorne AB, Logan RF, Hawkey CJ, et al. Randomised controlled trial of azathioprine withdrawal in ulcerative colitis. BMJ 1992;305:20-22.

20. Kennedy NA, Kalla R, Warner B, et al. Thiopurine withdrawal during sustained clinical remission in inflammatory bowel disease: relapse and recapture rates, with predictive factors in 237 patients. Aliment Pharmacol Ther 2014;40:1313-1323.

21. Moreno-Rincón E, Benítez JM, Serrano-Ruiz FJ, et al. Prognosis of patients with ulcerative colitis in sustained remission after thiopurines withdrawal. Inflamm Bowel Dis 2015;21:1564-1571. 